\title{
From Risk Factors to Detection and Intervention: A Metareview and Practical Proposal for Research on Cyberbullying
}

\author{
Andri IOANNOU ${ }^{1}$, Jeremy BLACKBURN ${ }^{2}$, Gianluca STRINGHINI ${ }^{3}$, \\ Emiliano DE CHRISTOFARO ${ }^{3}$, Nicolas KOURTELLIS ${ }^{2}$, \\ Michael SIRIVIANOS ${ }^{1}$, Panayiotis ZAPHIRIS ${ }^{1}$ \\ ${ }^{1}$ Cyprus University of Technology, 30 Archibishop Kyprianou Str., \\ 3036 Lemesos, 3036, Cyprus, Email: pzaphiri@cyprusinteractionlab.com \\ 2 Telefonica Research, Plaça Ernest Lluch i Martí, 5, 08019 Barcelona, Spain \\ ${ }^{3}$ University College London, Gower St, London WC1E 6BT, United Kingdom
}

\begin{abstract}
We sought to determine the level of maturity of the Cyberbullying research community and identify knowledge gaps that could be addressed in future projects in this area. Our findings suggest that the vast majority of academic contributions on cyberbullying focus on understanding the phenomenon, risk factors, and threats, with the prospect of suggesting possible protection strategies. The review calls for more research tackling the problem by leveraging statistical models and computational mechanisms geared to detect, intervene, and prevent cyberbullying. We argue that a multidisciplinary approach is needed for current challenges to be addressed and significant progress to be made, in order to combat this social menace.

Keywords: cyberbullying; internet bullying; cyber aggression, social media; metareview; literature survey; research agenda.
\end{abstract}

\section{Introduction}

The rapid evolution and use of online social networks (OSNs) and the ever-increasing number of adolescents admitting to have experienced cyberbullying, either as bullies, victims, or bystanders, creates an alarming need for advancing research and development in this area. A study by [1] surveyed a random sample of 457 students between the ages of 11 and 15 from a middle school in the Midwestern United States; approximately 34\% of the students reported experiencing cyberbullying in their lifetimes. An increase in cyberbullying can be noted, compared to previous results from the same authors [2] with a random sample of 4,441 students (aged 10-18) from 37 schools in a large district in the southern US; in this study $20 \%$ of the students had reported experiencing cyberbullying in their lifetimes. Furthermore, the Cyberbullying Research Center [3] studied the phenomenon in nine (random sampling) studies conducted between 2006 and 2015 to report that, on average, $26 \%$ of the students had been the victim of cyberbullying at some point in their lifetime, whilst $16 \%$ of them admitted they had cyberbullied others at some point in their lifetime. There is no doubt cyberbullying has become a social menace in the 21st century that needs to be addressed and combated.

\subsection{What is Cyberbullying}

Perhaps one of the most widely accepted definitions comes from [4] defining cyberbullying as "an aggressive intentional act carried out by a group or individual, using electronic forms of contact, repeatedly and over time against a victim who cannot easily defend him or herself” (p. 376). Generally speaking, cyberbullying can be seen as any form of abusive 
behavior in cyberspace. A typology of cyberbullying behavior has been proposed by Nocentini et al. [5] and includes four type of activity: written-verbal behavior (phone calls, text messages, e-mails, instant messaging, chats, blogs, social networking communities, websites), visual behavior (posting, sending or sharing compromising pictures and videos through mobile phone or internet), exclusion (purposefully excluding someone from an online group) and impersonation (stealing and revealing personal information, using another person's name and account). Research has demonstrated a number of negative effects of cyberbullying victimization including lower self-esteem, retaliating, and being scared, frustrated, angry and depressed [6]. When the victim fails to cope with the emotional tension of the abuse, the consequences of cyberbullying become even more serious leading to suicidal thoughts and behavior [7] [8].

\section{Objectives of Study and Methodology Used}

In order to understand scholarly activity on cyberbullying, we first compiled the corpus of research on cyberbullying using keyword search in scientific databases across disciplines -from social science and humanities to computer science. A taxonomy of the cyberbullying key-terms has been presented by [9] which includes the terms cyber-bullying, cybergrooming, cyber-stalking, electronic bullying, sms bullying, mobile bullying, online bullying, digital bullying, e-bullying, and Internet bullying. This taxonomy formed our basic keyword search, which was expanded to include more terms, considering the keywords and ideas presented in the manuscripts themselves, such as predator, victim, bystander, harassment, trolling, aggressive behavior, distressed behavior, hate speech, content monitoring, doxing, and cybermobbing. After a corpus of work was compiled, we attempted to synthesize the information presented in the manuscripts, organized in themes and research trajectories which follows. In the discussion section we elaborate on the open issues and potential directions for future research.

\section{Results}

\subsection{The Profiles of the Main Actors}

There are three main actors in the cyberbullying menace: the predator, the victim, and the bystander. A consistent profile of these actors has yet to be presented by researchers of cyberbullying and it appears to be a demanding area for exploration.

\subsubsection{Cyberbully}

In an overview of research on the types of cyberbullies (also cyberbullying perpetrators or predators), [10] identified five main categories, taking into account the psychological attributes (both personal and social) that underpin their behavior: the sociable cyberbully (cyberbullying for fun in order to entertain his/her friends without serious consideration of the victim's feelings); the lonely cyberbully (a relatively isolated cyberbully with no friends, spends his/her time by abusing others with whom s/he has little or no personal contact); the narcissistic cyberbully (a cyberbully demonstrating power by administering harm to another person); the sadistic cyberbully (a cyberbully enjoying causing distress, harm and suffering to another person); and the morally-driven cyberbully (a cyberbully feeling the victim is receiving justice for his/her actions). Additionally, in a survey research, Korean, You and Lim [11] used a sample of 3449 middle school students and demontrated a set of variables associated with more cyberbullying perpetration, particularly longer use of the Internet, more previous bullying and victim experiences, a higher aggression level, and lack of self-control. 


\subsubsection{Cybervictim}

Much of the previous research has attempted to identify risk factors for bullying and cyberbullying (typically examined together) focusing on demographic and behavioral measures. A number of psychological variables emerged in different studies describing the cybervictim. Sourander et al. [12] for example, found that cybervictim status was associated with living in a family with other than two biological parents, perceived emotional and peer problems, and feeling unsafe at school, whilst in Schneider et al. [8] cybervictims reported lower school performance and school attachment.

\subsubsection{Bystander}

The third actor in cyberbullying is the (negative) bystander who observes bullying without taking action. A comprehensive study of this actor comes from [13] who found that three factors increased the likelihood of negative bystander behavior: (i) the cyberspace, meaning that negative bystander behavior occurs more often in the cyberspace than offline, (ii) the private nature of the act, meaning that bystander behavior might occur more frequently in private forms of violence, and (iii) the experience of being a cyberbullying predator seems to be important predictor of negative bystander behavior. Two factors seem to decrease negative bystander behavior: (i) affective empathy i.e., the ability to understand the emotions of others, and (ii) cognitive empathy i.e., the ability to anticipate the consequences of one's actions on others [13].

\subsection{A Typology of Cyberbullying Actors}

A typology of cyberbullying actors in terms of their personal characteristics has yet to be presented in the cyberbullying literature. Instead, findings are sparse and inconsistent, calling for more work in this area.

\subsubsection{Gender}

Inconsistent findings have been reported regarding gender. Although research on traditional bullying shows that bullying is more common among boys (e.g., [14]), for cyberbullying, findings are mixed. Several studies report that males are indeed more likely to be cyberbullies than their female counterparts [15] [16]; yet, others report no gender differences [17] and others claim that girls outnumber boys [18] [19]. In a study of 2186 middle and high school students, [20] explored the factors that contribute to engagement with cyberbullying, looking at groups of actors including victims, bullies, bully-victims (both bully and be bullied online) and those not involved. Findings included that bullyvictims were more likely to be females, whilst no gender differences were found among students who bullied others or who were victimized online. Moreover, in Schneider et al. [8], a total of 20406 students (9-12 grade) in MetroWest Massachusetts completed surveys assessing their bullying victimization and psychological distress. The study showed that girls were more likely, than were boys, to report cyberbullying as predators, especially in combination with school bullying, but they were also more likely to be victims of both types of bullying (on school property and cyber).

\subsubsection{Age}

While traditional bullying seems to peak during middle school, cyberbullying peaks somewhat later [21]. In fact, with age, there seems to be a gradual shift away from traditional forms of bullying such as spreading rumours, to cyberbullying; for example, [20] found that the older the student, the more likely s/he was to cyberbully others or to both bully and be bullied online, than to be neither a bully nor victim. It should be noted that most cyberbullying research conducted so far has focused on adolescents [22], while very little cyberbullying research exists on primary school students [23]. 


\subsubsection{Sexual Orientation}

There seems to be a consistent finding that non-heterosexual individual are targets of traditional bullying and cyberbullying. For example, [8] reported that non-heterosexually youths were more likely to be victims of cyberbullying, compared to heterosexual $(10.5 \%$ vs $6.0 \%$ ). A previous study reported similar findings, showings that lesbian, gay, bisexual, and transsexual individuals were twice as likely to experience cyberstalking or e-mail harassment from a stranger, compared to heterosexual individuals.

\subsubsection{Other Personal Characteristics}

A few other profile patterns of cyberbullying actors have been reported in the literature as follows:

(i) Computer use. Students who were victims, bullies, and bully-victims were more likely than students who were not involved in cyberbullying to use the computer for more hours a day and to give their password to friends [20]. Also, intensive use of the Internet emerged as a risk factor for child cyber-harassment [24]. Furthermore, the location of the computer at home was found to be a predictive factor of cyber victimization. Children who use the computer in private places at their home (e.g., bedroom) were at higher risk to be victimized than children who used computers in a public space in their home [25].

(ii) School performance. According to [8], youth who reported lower school performance and lower school attachment were also more likely to be victimized with cyberbullying; in particular, students who received mostly Ds and Fs were twice as likely to be victims of cyberbullying compared to students who received mostly As (11.3\% vs 5.2\%).

(iii)Bullied person, bullying others. There is a lack of research on the bully-victim group (persons being bullied and also bullying others) whilst a potential causal link is alarming and warrants further investigation (Does bullied person become a bully?). For example, in their survey research, [11] found that previous offline bullying and victim experiences were associated with more cyberbullying. Similarly, Mishna et al. [20] argued that the cyber offers easy space for "revenge" or "payback" with high prevalence of bully-victim behavior (26\%) in a sample of 2186 participants [20].

\subsection{The Problematic Nature of the Label "cyberbullying”}

Defining, measuring, or detecting specific cyberbullying behavior is not a trivial task. Existing definitions of cyberbullying, as well as the one adhered in this review by [4], often incorporate the criteria of traditional bullying such as repetition over time and imbalance of power (a victim who cannot easily defend him/herself). However, due to the unique nature of cyber-based communication, it is difficult to identify such criteria in the cyber abuse [26]. As a result, there is uncertainty regarding the operational definition of cyberbullying and how to effectively measure it [26]. On that note, [27] argued that most works on cyberbullying as claimed by the original authors, are in fact more accurately described as research on cyberaggression, as they do not take into account the frequency of the event and the imbalance of power. Below we discuss three relevant dimensions that, if further explored, should inform the operational definition and thus, measurement and detection of cyberbullying.

\subsubsection{The Power Dimension}

Although a central aspect of most operational definitions of traditional bullying, "power" is difficult to determine in the cyber context [26]. Is "power" the ability to remain anonymous in the cyberspace [4]? Is it the ability to demonstrate superior technological knowledge [28] ? Is it the immediacy of content dissemination and capacity to humiliate on a grand scale [29] ? Or is "power" the perceived popularity of the predator causing more psychological 
distress? The later was investigated by [30] who found that, compared to being harassed by an unpopular cyberbully, being harassed by a popular cyberbully was more distressing and elicited more negative mood and helplessness. Understanding what "power" in cyberspace entails will significantly inform the operational definition of cyberbullying.

\subsubsection{The Dimension of Severity -- Duration and Level of Insult}

Cyberbullying can occur anytime and anywhere and is believed to be more damaging than traditional face-to-face bullying because of the fluidity and frequency of the bullying behavior using technology. In fact, several authors [29][31] hypothesised that because bullying acts performed online are visible for a long(er) period of time and to a large audience (who may also join the bully), their negative effects can be more severe and longer lasting, compared to victims of repeated (offline) bullying acts. Yet, there is lack of empirical research tackling cyberbullying as a sequence of actions that involve repetition of harming content and levels of severity. Potha and Maragoudakis [32] seem to be the first to have considered the duration and level of insult in cyberbullying; using a dynamic time warping algorithm, they were able to provide an immediate indicator for the severity of cyberbullying within a given dialogue. Yet, more research is needed for understanding (and detecting) the level of insult and duration of the cyber abuse.

\subsubsection{The Anonymity Dimension}

The anonymity in cyberbullying adds a totally new dimension to the nature of traditional bullying. The devices that are used (such as mobile phones and computers) make it easier for a perpetrator to act anonymously (e.g., by using a nickname) and without directly facing the victim [33]. Moreover, cyberbullies have less chance of getting caught or punished as they can perpetrate without adult supervision [4]. What makes the anonymity dimension more apparent in cyberbullying, is the evidence that many cyberbullies do not choose inperson bullying if the cyber route is denied. As [34] discussed, cyberbullies might not bully in person because they feel powerless socially or because they are invested in school and academics, but are willing to bully online because they believe that cyberbullying is without risk since adults are not present.

\section{Implications and Recommendations}

This review explores research pertaining to cyberbullying across disciplines, from social science and Humanities to computer science. It appears, research on cyberbullying is still at its infancy and most studies are mere reports of prevalence rates and relationships among variables. There is less work on intelligence techniques for the detection of cyberbullying when it occurs, a rather demanding area for exploration. The following recommendations have been developed through our analyses, aiming to guide further research in the field. All of them call for a multidisciplinary approach that integrates knowledge from the humanities and computer science in order to address current and future research challenges.

1. Conduct more natural experiments. Efforts from the social sciences mainly focus on the participants' perceived experiences of being engaged in cyberbullying as predators, victims, or bystanders; less often, researchers rely on experimental conditions studying cyberbystander behavior only. This research is definitely of value, yet natural experiments with real-life OSNs data are needed to track and understand the behavior of predators and their victims in space and time. Real cyberbullying activity abounds in today's cyberspace. Synergies between researchers in humanities and computers science will help gather and analyse these datasets in order to advance the study of this social menace.

2. Bring more factors into the equation. Factors such as severity (level of insult and duration) and power are yet to be considered in cyberbullying research, beyond mere 
discussion of their importance in social sciences research. Valuable insight could emerge from research that tackles whole blocks of cyberbullying activity in OSNs, demonstrating and understanding the evolution, repetition, and imbalance of power evident in these events. Current algorithms seem to detect the problem only up to some degree of success; we would argue that consideration of these factors might improve their intelligence.

3. Juxtapose cyberbullying patterns across platforms. Cyberbullying is not everywhere. Different OSNs platforms might encourage or inhibit cyberbullying for different reasons. For example, it is possible that the severity of cyberbullying is linked to the degree of platform anonymity. Today's easy access to data from various online social media platforms (e.g. instagram, 4chan, Twitter, TwitchTV, Facebook, snapchat) allows researchers to juxtapose patterns of behavior and thus, consider causal relationships between factors such as (platform) anonymity and severity of cyberbullying.

4. Backtrack cyberbullying behavior. There is limited research on how cyberbullying begins, is fed, and evolves. There is also limited research on the bully-victim as a separate actor of cyberbullying, although an alarming consideration, emerging from social sciences research, is that a bullied person becomes a bully. Computer science algorithms can help backtrack cyberbullying behavior looking at these issues e.g., when and how one engages in bullying (e.g., person acting properly, then bullies after being bullied).

5. Consider a multi-cultural approach. The typology of cyberbullying participants in terms of gender, age, sexual orientation and other characteristics should involve cultural elements, which might be the cause of inconsistent research findings in regards to these factors. Similarly, cyberbullying actors -- predator, victim, and bystander -- might possess different profiles across cultures. A multi-cultural approach to cyberbullying could generate further useful insight into advancing these typologies. Here under represented communities in such studies (e.g. Africa communities) should be included. This appears to be a demanding area for exploration in the social sciences, which can greatly inform algorithmic approaches to detection and prevention from a computer science perspective.

6. Begin the dialog between social science/ humanities and computer science. Communication between disciplines is virtually non-existent within the cyberbullying arena.

\section{Conclusion}

While there is an unceasing flow of media stories reporting cases of cyberbullying, particularly within online social media, research efforts in the academic community are scattered over different topics and across the humanities and computer science. The majority of academic contributions focus on understanding the phenomenon, risk factors and threats with the prospect of suggesting possible protection strategies. Detecting cyberbullying when it occurs and identifying predators and their victims in real computermediated communication remains an open issue to be solved, before intervention and prevention methods can be addressed. Recognizing blocks of cyberbullying activity and understanding dimensions such as duration, severity, power, and anonymity can shed valuable insight into how cyberbullying is fed and evolves. There is an immediate need for true multidisciplinary work between social and computer sciences, in order for current challenges to be effectively addressed and significant progress to be made, and we are confident that this review will serve as a multidisciplinary agenda to guide future research in this area.

Africa is an under represented continent in such studies. Studies that document cyberbullying in Africa, ways of combatting it in such communities and mechanisms for 
encouraging training and awareness for young communities on online behavior and protection.

\section{Acknowledgements}

This research has been fully funded by the European Commission as part of the ENCASE project (H2020-MSCA-RISE of the European Union under GA number 691025).

\section{References}

[1] Hinduja, S., \& Patchin, J. W. (2015). Cyberbullying legislation and case law: Implications for school policy and practice. Cyberbullying Research Center.

[2] Hinduja, S., \& Patchin, J. W. (2013). Social influences on cyberbullying behaviors among middle and high school students. Journal of youth and adolescence, 42(5), 711-722.

[3] Cyberbullying Research Center (2015). Summary of Our Cyberbullying Research (2004-2015). Retrieved July 2015, from http://cyberbullying.org/summary-of-our-cyberbullying-research

[4] Smith, P. K., Mahdavi, J., Carvalho, M., Fisher, S., Russell, S., \& Tippett, N. (2008). Cyberbullying: Its nature and impact in secondary school pupils. Journal of child psychology and psychiatry, 49(4), 376-385.

[5] Nocentini, A., Calmaestra, J., Schultze-Krumbholz, A., Scheithauer, H., Ortega, R., \& Menesini, E. (2010). Cyberbullying: Labels, behaviours and definition in three European countries. Australian Journal of Guidance and Counselling, 20(02), 129-142.

[6] Smith-Ross, C., Esmail, A., Omar, A., \& Franklin, K. (2014). Chapter Nine Bullying: Recognizing the Warning Signs. Alleviating bullying: Conquering the challenge of violent crimes, 146.

[7] Foody, M., Samara, M., \& Carlbring, P. (2015). A review of cyberbullying and suggestions for online psychological therapy. Internet Interventions, 2(3), 235-242.

[8] Schneider, S. K., O'Donnell, L., Stueve, A., \& Coulter, R. W. (2012). Cyberbullying, school bullying, and psychological distress: A regional census of high school students. American Journal of Public Health, 102(1), 171-177.

[9] Al Mazari, A. (2013). Cyber-bullying taxonomies: Definition, forms, consequences and mitigation strategies. 5th International Conference on Computer Science and Information Technology (CSIT) (pp. 126133). IEEE.

[10] Kyriacou, C., \& Zuin, A. (2016). Cyberbullying and moral disengagement: an analysis based on a social pedagogy of pastoral care in schools. Pastoral Care in Education, 34(1), 34-42.

[11] You, S., \& Lim, S. A. (2016). Longitudinal predictors of cyberbullying perpetration: Evidence from Korean middle school students. Personality and Individual Differences, 89, 172-176.

[12] Sourander, A., Klomek, A. B., Ikonen, M., Lindroos, J., Luntamo, T., Koskelainen, M., . . Helenius, H. (2010). Psychosocial risk factors associated with cyberbullying among adolescents: A population-based study. Archives of general psychiatry, 67(7), 720-728.

[13] Barlinska, J., Szuster, A., \& Winiewski, M. (2013). Cyberbullying among adolescent bystanders: Role of the communication medium, form of violence, and empathy. Journal of Community \& Applied Social Psychology, 23(1), 37-51.

[14] Sanchez, F. C., Romero, M. F., Navarro-Zaragoza, J., Ruiz-Cabello, A. L., Frantzisko, O. R., \& Maldonado, A. L. (2016). Prevalence and patterns of traditional bullying victimization and cyber-teasing among college population in Spain. BMC public health, 16(1), 1.

[15] Li, Q. (2006). Cyberbullying in schools a research of gender differences. School psychology international, 27(2), 157-170.

[16] Lapidot-Lefler, N., \& Dolev-Cohen, M. (2015). Comparing cyberbullying and school bullying among school students: prevalence, gender, and grade level differences. Social psychology of education, 18(1), 1-16.

[17] Patchin, J. W., \& Hinduja, S. (2006). Bullies move beyond the schoolyard a preliminary look at cyberbullying. Youth violence and juvenile justice, 4(2), 148-169.

[18] Keith, S., \& Martin, M. E. (2005). Cyber-bullying: Creating a culture of respect in a cyber world. Reclaiming children and youth, 13(4), 224.

[19] Kowalski, R. M., \& Limber, S. P. (2007). Electronic bullying among middle school students. Journal of adolescent health, 41(6), S22--S30.

[20] Mishna, F., Khoury-Kassabri, M., Gadalla, T., \& Daciuk, J. (2012). Risk factors for involvement in cyber bullying: Victims, bullies and bully--victims. Children and Youth Services Review, 34(1), 63-70.

[21] Pabian, S., \& Vandebosch, H. (2016). An investigation of short-term longitudinal associations between social anxiety and victimization and perpetration of traditional bullying and cyberbullying. Journal of youth and adolescence, 45(2), 328-339. 
[22] Steffgen, G., Konig, A., Pfetsch, J., \& Melzer, A. (2011). Are cyberbullies less empathic? Adolescents' cyberbullying behavior and empathic responsiveness. Cyberpsychology, Behavior, and Social Networking, 14(11), 643-648.

[23] Tangen, D., \& Campbell, M. (2010). Cyberbullying Prevention: One Primary School's Approach. Australian Journal of Guidance and Counselling, 20(02), 225-234.

[24] Wolak, J., Mitchell, K. J., \& Finkelhor, D. (2007). Does online harassment constitute bullying? An exploration of online harassment by known peers and online-only contacts. Journal of adolescent health, 41(6), S51--S58.

[25] Sengupta, A., \& Chaudhuri, A. (2011). Are social networking sites a source of online harassment for teens? Evidence from survey data. Children and Youth Services Review, 33(2), 284-290.

[26] Corcoran, L., Guckin, C. M., \& Prentice, G. (2015). Cyberbullying or cyber aggression?: A review of existing definitions of cyber-based peer-to-peer aggression. Societies, 5(2), 245-255.

[27] Hosseinmardi, H., Mattson, S. A., Rafiq, R. I., Han, R., Lv, Q., \& Mishra, S. (2015). Detection of cyberbullying incidents on the instagram social network. arXiv preprint arXiv:1503.03909.

[28] Vandebosch, H., \& Van Cleemput, K. (2009). Cyberbullying among youngsters: Profiles of bullies and victims. New media \& society, 11(8), 1349-1371.

[29] Langos, C. (2012). Cyberbullying: The challenge to define. Cyberpsychology, Behavior, and Social Networking, 15(6), 285-289.

[30] Pieschl, S., Porsch, T., Kahl, T., \& Klockenbusch, R. (2013). Relevant dimensions of cyberbullyingResults from two experimental studies. Journal of Applied Developmental Psychology, 34(5), 241-252.

[31] Sticca, F., \& Perren, S. (2013). Is cyberbullying worse than traditional bullying? Examining the differential roles of medium, publicity, and anonymity for the perceived severity of bullying. Journal of youth and adolescence, 42(5), 739-750.

[32] Potha, N., \& Maragoudakis, M. (2014). Cyberbullying detection using time series modeling. 2014 IEEE International Conference on Data Mining Workshop, (pp. 373-382).

[33] Slonje, R., Smith, P. K., \& Frisen, A. (2013). The nature of cyberbullying, and strategies for prevention. Computers in Human Behavior, 29(1), 26-32.

[34] Englander, E. (2012). Spinning our wheels: Improving our ability to respond to bullying and cyberbullying. Child \& Adolescent Psychiatric Clinics of North America. 